\title{
Ophthalmological Screening for von Hippel-Lindau Disease
}

\author{
A. T. MOORE*, E. R. MAHER**, P. ROSEN, $\dagger$, Z. GREGOR $\dagger$, A. C. BIRD \\ Cambridge and London
}

\begin{abstract}
Summary
Forty seven individuals (from 16 kindreds) without prior evidence of retinal haemangiomas underwent full ophthalmological assessment as part of a comprehensive screening programme for Von Hippel-Lindau disease. Ten were known to be affected on the basis of non-ocular involvement and 37 were at $50 \%$ risk of having the abnormal gene. Seventeen angiomas were detected in 14 individuals (six affected patients and eight at risk relatives). Regular detailed ophthalmological assessment is important for all patients with von Hippel-Lindau disease and for relatives at risk of inheriting the abnormal gene.
\end{abstract}

Von Hippel-Lindau (VHL) disease is an autosomal dominant disorder with variable expression. The cardinal features are retinal haemangiomas, central nervous system haemangioblastomas, renal cell carcinoma, phaeochromocytoma, and pancreatic, renal and epididymal cysts. ${ }^{1-5}$ Retinal angiomatosis is the most frequent initial manifestation and develops in more than $70 \%$ of patients by age 60 years. ${ }^{4}$ Retinal angiomas are most commonly seen in the peripheral retina but may also occur on the optic disc or at the ora serrata. Although patients may present with visual symptoms, small angiomas are often asymptomatic and careful ophthalmoscopic screening of affected individuals and those family members at risk of inheriting the VHL gene mutation is necessary to detect ocular involvement. Early detection of these retinal angiomas is important for genetic counselling ${ }^{4,5,6}$ and improves the visual prognosis by allowing early treatment ${ }^{7,8}$. Other central ner- vous system or visceral manifestations can also be detected at an early pre-symptomatic stage by screening investigations ${ }^{4}$.

A systematic protocol has been developed for the screening and review of affected patients and relatives at risk of having the abnormal gene. ${ }^{4}$ We report in detail the results of the ophthalmological screening protocol in our clinical practice.

\section{Patients and Methods}

Forty seven individuals (from 16 kindreds) without prior evidence of retinal haemangiomas underwent full ophthalmological assessment as part of a comprehensive screening programme for VHL. (Table I). Ten were known to be affected by VHL on the basis of non-ophthalmic manifestations of the disorder (mean age $+/-$ SD $40.8+/-13.0$, range 19-62 years), and 37 were asymptomatic relatives at $50 \%$ risk of inheriting the abnormal

From Departments of Ophthalmology* and Clinical Genetics** Addenbrooke's Hospital, Cambridge and Moorfields Eye Hospital† London.

Correspondence to: Mr A. T. Moore, FRCS, Eye Department, Addenbrookes Hospital, Hills Road, Cambridge CB1 2QQ. 
Table 1. Cambridge screening protocol for von Hippel-Lindau disease in affected patients and at risk relatives. (Full details in Maher et al., 1990)

\section{Affected Patient}

1. Annual physical examination and urine testing.

2. Annual ophthalmoscopy and fluoroscein angioscopy (see text).

3. MRI (or CT) brain scan every 3 years to age 50 and every 5 years thereafter.

4. Annual renal ultrasound scan, with CT scan every 3 years (more frequently if multiple renal cysts present).

5. Annual 24 hour urine collection for VMAs.

\section{At Risk Relative}

1. Annual physical examination and urine testing.

2. Annual ophthalmoscopy from age 5 to 60 years, and fluoroscein angioscopy from age 10 (see text).

3. MRI (or CT) brain scan every 3 years to from age 15 to 40 years and then every 5 years until age 60 years.

4. Annual renal ultrasound scan, with abdominal CT scan every 3 years from age 20 to 65 years.

5. Annual 24 hour urine collection for VMAs.

N.B. These guidelines are for asymptomatic individuals, symptomatic patients should be investigated urgently. The frequency of screening may be reduced in relatives in whom DNA linkage studies reduce their risk.

gene (mean age $+/-$ SD $28.8+/-16.4$, range 5-68 years).

Direct and indirect ophthalmoscopy was performed in all patients. If an angioma was seen formal fluorescein angiography was performed to document the site and size of the lesion. Fluorescein angioscopy was performed in patients with normal ophthalmoscopy. Five mls of $20 \%$ sodium fluorescein were injected as an intravenous bolus and the fundus was viewed through the blue excitation filter of the indirect ophthalmoscope; no barrier filter was used. Any angiomas found on angioscopy were subsequently documented using colour photography. Systemic screening was carried out according to the Cambridge protocol. ${ }^{4}$ (Table I)

\section{Results}

The results of ophthalmological examination are shown in Figure 1. Four of 10 affected individuals and seven of 37 asymptomatic relatives had retinal angiomas visible on ophthalmoscopy (Figs 2, 3, 4). Thirty two individuals with a normal fundus examination (six of whom were known to have the abnormal gene) underwent fluorescein angioscopy (the procedure was omitted in four young children with normal ophthalmoscopy). Small angiomas were detected in three patients (Fig. 5). One of these patients showed generalised vascular leakage and capillary abnormalities in the peripheral retina of one eye unrelated to the retinal angioma. A similar retino-vascular abnormality was seen in the inferior retina in one other asymptomatic relative who showed no other evidence of VHL disease on further investigation. Overall, 17 retinal angiomas were detected in 14 patients, two of whom had bilateral involvement. Eleven angiomas were in the peripheral retina, five on the optic disc and one at the ora serrata. Retinal exudates were present in one eye and another with a large optic disc haemangioma had an epiretinal membrane involving the macula (Fig. 2). Of 37 relatives at $50 \%$ risk of inheriting the VHL disease mutation 11 were found to be affected as a result of ophthalmoscopic and systemic screening.

\section{Discussion}

Retinal haemangiomas are the commonest manifestation of VHL disease and are often the earliest sign of the disease. ${ }^{4}$ Large angiomas are seen as raised red oval tumours usually in the mid peripheral retina with a dilated feeding artery and draining vein (Fig. 3). Such lesions often give rise to symptoms related to retinal detachment or macular oedema and are easily recognised on ophthalmoscopy. Small peripheral angiomas however give rise to no symptoms and may be difficult to see on ophthalmoscopy. We have found fluorescein angioscopy using the indirect opthalmoscope to be extremely useful in identifying small angiomas which may otherwise have been undetected (Fig. 5). These small lesions consisted of small flat tortuous arteriovenous malformations which leaked fluorescein on angiography. Two individuals (one affected and one at risk) showed evidence of leakage of fluorescein from peripheral retinal veins, and in one there was evidence of associated microvascular abnormalities without angioma formation. We did not observe any microvascular abnormalities similar to microaneurysms that were reported by Salazar and Lamiell $^{6}$. In this small series optic disc haemangiomas were commoner than previously 


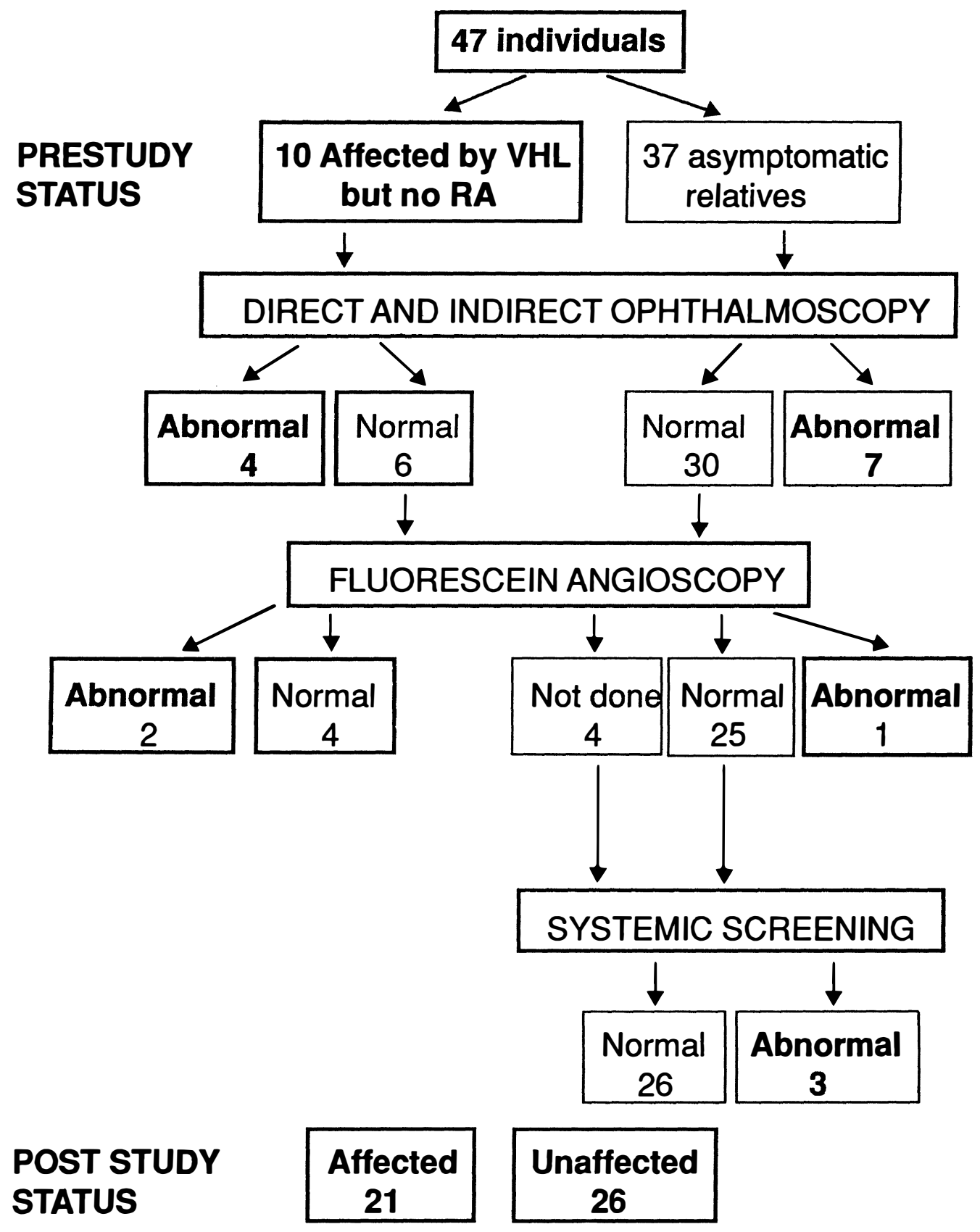

Fig. 1. Results of ophthalmoscopic and systemic screening in patients with von Hippel-Lindau disease and relatives at $50 \%$ risk of inheriting the abnormal gene. 


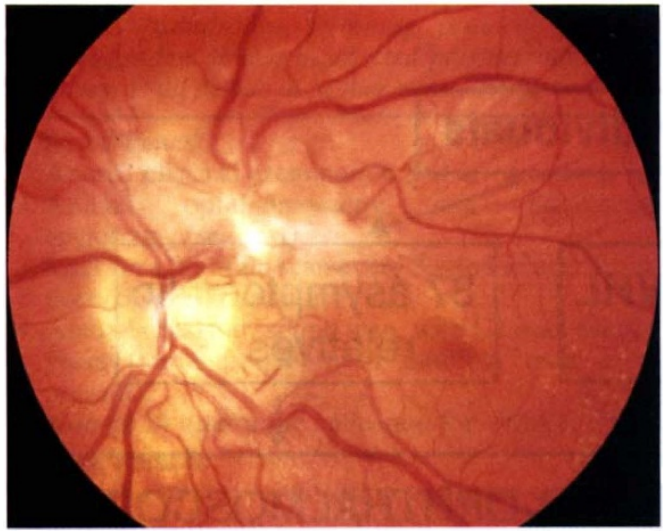

Fig. 2a.

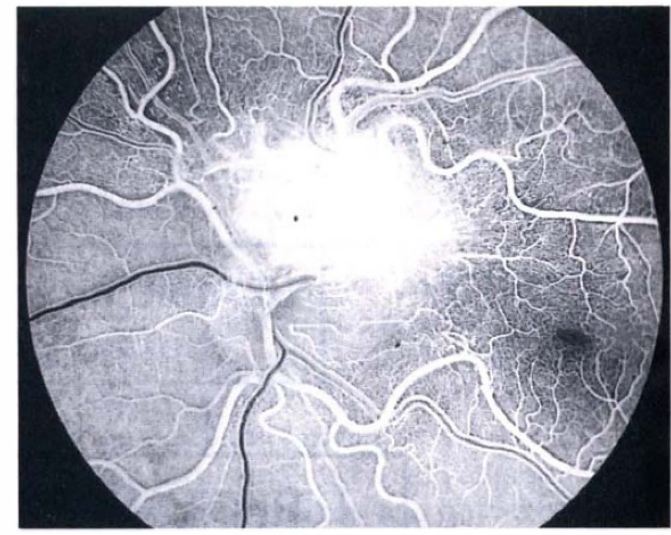

Fig. 2l).

Fig. 2 Colour photograph and fluorescein angiogram of an optic disc haemangioma in an at risk relative.

reported. This may reflect selection of patients not known to have retinal angiomas since small optic disc haemangiomas are usually asymptomatic and if they are very slow growing they would not give rise to symptoms for many years.

Treatment is straightforward and the complications of photocoagulation few if lesions are small ${ }^{8}$, but in the presence of large lesions vision may be irrecoverable ${ }^{7,8}$. This implies that lesions should be identified early for ideal management. Although we have demonstrated that ophthalmoscopy is sufficient to

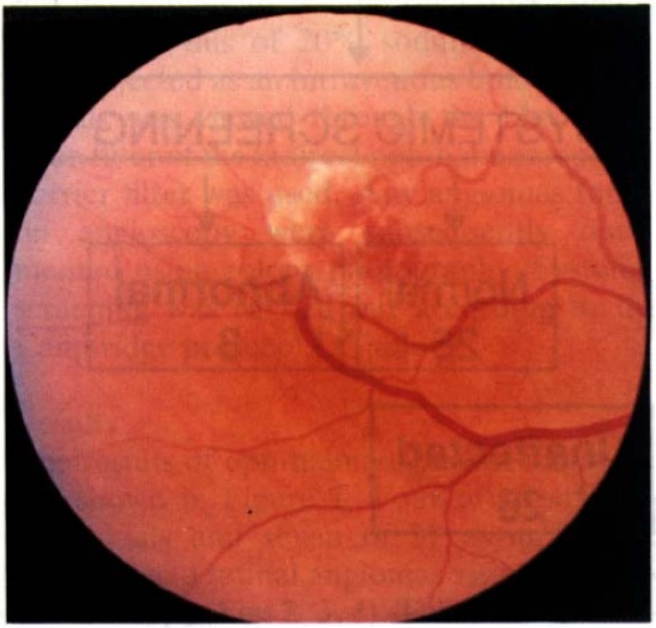

Fig. 3a. identify most angiomas, some may grow rapidly $^{8}$ and we believe it is justifiable to use fluorescein angioscopy, which is a routine procedure with few side effects ${ }^{9}$, to detect small angiomas that may be missed on ophthalmoscopy. Although most angiomas are first detected in the second and third decades of life ${ }^{3-5}$ the age of onset is very variable; retinal angiomas have been reported in infants and young children, ${ }^{5}$ and may present for the first time in patients over the age of 80 years (personal observation). Individuals known to have the abnormal gene or who are at $50 \%$

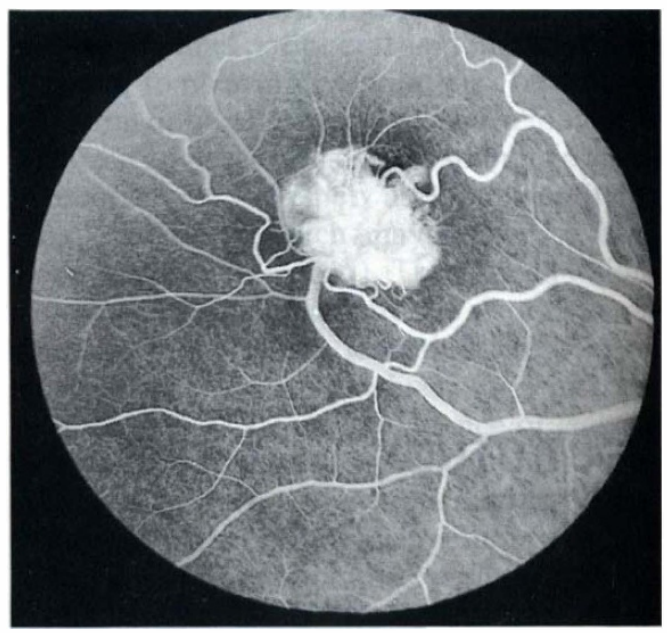

Fig. 3b.

Fig. 3. Colour photograph and fluorescein angiogram of typical peripheral angioma in an affected patient. 


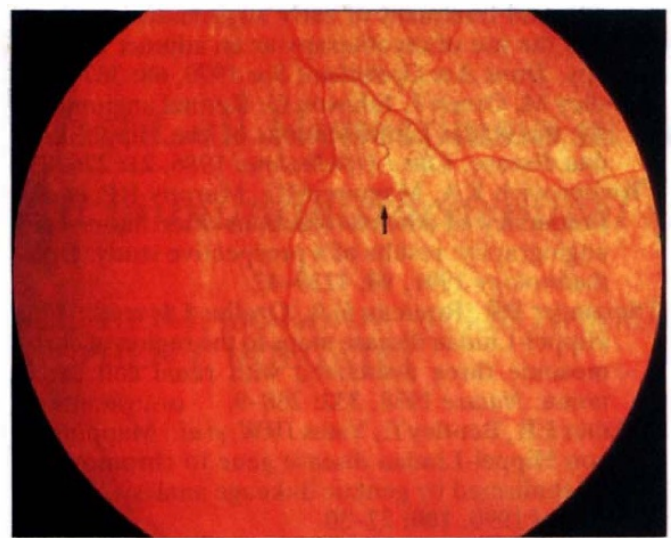

Fig. 4. Colour photograph of a small peripheral retinal haemangioma (arrow).

risk of inheriting the VHL mutation should therefore be examined regularly from early childhood.

The second objective of ophthalmic examination is to detect the abnormal phenotype as a marker for the abnormal gene. The presence of any manifestation of the disease identifies that individual as being affected, at risk of having affected descendants, and of developing complications which may be life threatening. The detection of any angioma however small is significant in this regard. That fluorescein angiography appears to increase the sensitivity of the examination justifies its use in those subjects at risk of having the abnormal gene but in whom there are no

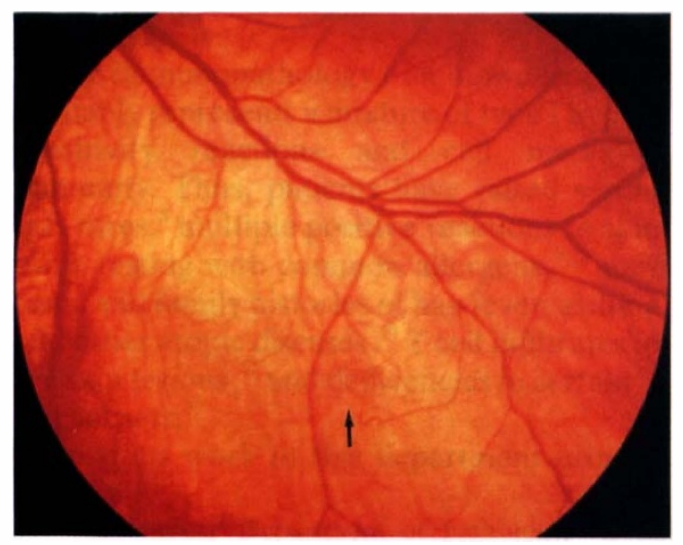

Fig. 5 a. other manifestations of disease. It is the ophthalmologist who will detect the abnormal phenotype in the majority of cases and he should initiate the appropriate genetic and systemic screening investigations for all affected individuals and at risk family members. Close cooperation with clinical geneticists and other specialists is essential.

The gene for von Hippel-Lindau disease has recently been mapped to the short arm of chromosome three $\mathrm{e}^{10-12}$ and DNA markers which flank the VHL disease locus have recently been identified. ${ }^{12}$ Diagnosis using linked DNA markers will allow relatives shown to be at low risk to be screened less frequently so concentrating resources on affected individuals and high risk relatives. The number of families in which pre-symptomatic diagnosis is now possible is limited by absence of stored DNA from affected family members who have died, and the informativeness of currently available DNA markers. Ultimately the isolation of the VHL disease gene will allow accurate documentation of the distribution of the abnormal gene in families in which the mutation has been characterised. In the meantime DNA should be stored from all affected individuals and first degree relatives.

We are grateful to Katherine Haslam for carrying out the fundus photography and fluorescein angiography.

Key words: Fluorescein angioscopy, retinal haemangioma, screening, Von Hippel-Lindau disease.

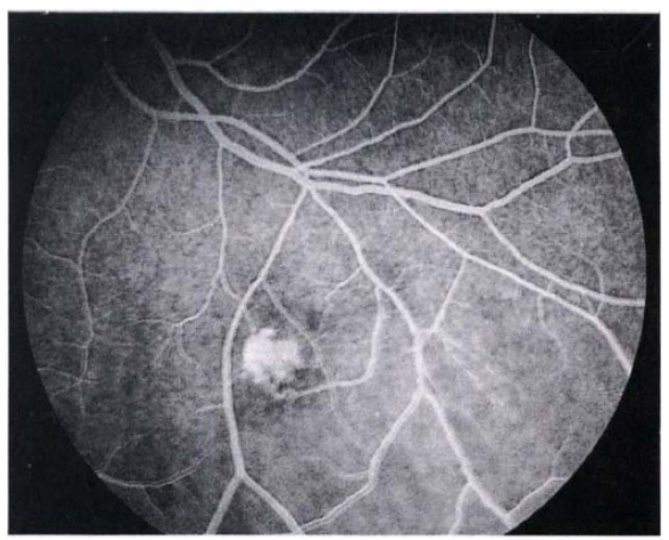

Fig. 5h.

Fig. 5. Colour photograph and fluorescein angiogram of small peripheral angioma (arrow) identified on fluorescein angioscopy. 


\section{References}

${ }^{1}$ Melmon K L and Rosen SW: Lindau's disease; review of the literature and study of a large kindred. Am J Med 1964, 36: 595-617.

${ }^{2}$ Horton WA, Wong V, Eldridge R: Von HippelLindau disease. Clinical and pathological manifestations in nine families with 50 affected members. Arch Int Med 1976, 136: 769-77.

${ }^{3}$ Lamiell JM, Salazar FG, Hsia YE: Von HippelLindau disease affecting 43 members of a single kindred. Medicine 1989, 68: 1-29.

${ }^{4}$ Maher ER, Yates JRW, Harries R, et al.: Clinical features and natural history of von Hippel-Lindau disease. Quart J Med 1990, 77: 1151-63.

${ }^{5}$ Green JS, Bowmer MI, Johnson GJ: von Hippel Lindau disease in a Newfoundland kindred. Can Med Assoc J 1986, 134: 133-46.

${ }^{6}$ Salazar FG and Lamiell JM: Early identification of retinal angiomas in a large kindred with Von Hippel-Lindau disease. Am J Ophthalmol 1980, 89: 540-5.

${ }^{7}$ Welch RB: Von Hippel Lindau disease: the recogni- tion and treatment of early angiomatosis retinae and the use of cryotherapy as an adjunct to therapy. Trans Am Opthalmol Soc 1970, 68: 367-423.

${ }^{8}$ Ridley M, Green J, Johnson G: Retinal angiomatosis: the ocular manifestations of von Hippel-Lindau disease. Can J Ophthalmol 1986, 21: 276-83.

${ }^{9}$ Kwiterovich KA, Maguire MG, Murphy RP, et al.: Frequency of advęrse reactions after fluorescein angiography: results of a prospective study. $\mathrm{Oph}$ thalmology 1991, 98: 1139-42.

${ }^{10}$ Seizinger BR, Rouleau GA, Ozelius LJ, et al.: Von Hippel-Lindau disease maps to the region of chromosome three associated with renal cell carcinoma. Nature 1988, 332: 268-9.

${ }^{11}$ Maher ER, Bentley E, Yates JRW, et al.: Mapping of von Hippel-Lindau disease gene to chromosome 3 p confirmed by genetic linkeage analysis. $J \mathrm{Neu}$ rol Sci 1990, 100: 27-30.

${ }^{12}$ Maher ER, Bentley E, Yates JRW, et al:: Localization of the gene for von Hippel Lindau disease to a small region of chromosome 3 confirmed by genetic linkeage analysis. Genomics 1991, 10: 957-60. 